\title{
Effects of aliphatic dioic acids and glycerol-1,2,3-tris(dodecanedioate) on D-glucose-stimulated insulin release in rat pancreatic islets
}

\author{
W. J. Malaisse ${ }^{1 *}$, A. V. Greco ${ }^{2}$, and G. Mingrone ${ }^{2}$ \\ ${ }^{1}$ Laboratory of Experimental Medicine, Brussels Free University, Brussels, Belgium \\ ${ }^{2}$ Instituto de Clinica Medica, Universita Cattolica del Sacro Cuore, Rome, Italy
}

(Received 9 August 1999 - Revised 24 February 2000 - Accepted 9 March 2000)

\begin{abstract}
Aliphatic dioic acids have been proposed as alternative nutrients in selected clinical situations. In this study, their possible insulinotropic action was investigated in isolated rat pancreatic islets prepared from fed rats. Azelaic acid, sebacic acid and tridecanedioic acids, when tested at a $10.0 \mathrm{mM}$ concentration, were found to augment insulin release evoked by D-glucose $(7.0 \mathrm{mM})$ in the pancreatic islets. Likewise, glycerol-1,2,3-tris(dodecanoedioate), when used at concentrations close to $1.0 \mathrm{mM}$, increased the secretory response to the hexose. It is speculated that these findings may extend to insulin-producing cells, the knowledge that aliphatic dioic acids or their esters may act as energy substrates, e.g. in parenteral nutrition.
\end{abstract}

Pancreatic islets: Insulin secretion: Aliphatic dioic acids

Medium-chain dicarboxylic acids have been recently regarded as possible alternate fuel substrates both in normal and pathological conditions in man (Mingrone et al. 1989, 1992, 1996; Tacchino et al. 1990; Bertuzzi et al. 1991). The energy densities of these compounds $(20.79 \mathrm{~kJ} / \mathrm{g}(4.97 \mathrm{kcal} /$ g) for azelaic acid, C9, $27.78 \mathrm{~kJ} / \mathrm{g}(6.64 \mathrm{kcal} / \mathrm{g})$ for sebacic acid, C10, and $30.12 \mathrm{~kJ} / \mathrm{g}(7.2 \mathrm{kcal} / \mathrm{g})$ for dodecanedioic acid, C12) are approximately in-between the values known for the conventional energy substrates, such as glucose $(15.69 \mathrm{~kJ} / \mathrm{g}(3.75 \mathrm{kcal} / \mathrm{g}))$ and fatty acids $(37.67 \mathrm{~kJ} / \mathrm{g}$ $(9 \mathrm{kcal} / \mathrm{g}))$. A major drawback of these dioic acids is the relatively high degree of urinary loss which is proportionally lower as their chain length increases (Bertuzzi et al. 1991; Mingrone et al. 1996). The triacylglycerol of dodecanedioic acid, however, while preserving its water solubility appears to have the lowest urinary excretion in experimental animals, corresponding to approximately $0.67 \%$ of the administered amount. Kinetics studies in rats (De Gaetano et al. 1999) have shown that this triacylglycerol has a large volume of distribution (approximately $0.5 \mathrm{l} / \mathrm{kg}$ body weight) with a fast disappearance rate from plasma $\left(0.42 \mathrm{~min}^{-1}\right)$, whereas one of its product of hydrolysis, dodecanedioic acid, has a very small volume of distribution (approximately $0.041 / \mathrm{kg}$ body weight) and a high tissue uptake with maximal transport rate of $0.636 \mathrm{mmol} / \mathrm{min}$. The measured dodecanedioic acid tissue uptake rate is consistent with the possibility of achieving substantial energy delivery, should it be added to parenteral nutrition formulations. Finally, the amount of $\mathrm{Na}$ administered with the triacylglycerol form is one-half of that necessary for water solubility with the free diacid.
It has been demonstrated, by using euglycaemic hyperinsulinaemic clamp and indirect calorimetry, that medium-chain dicarboxylic acids can compete with glucose for cellular utilization in human subjects (Raguso et al. 1994; Mingrone et al. 1997). A direct effect of these acids on pancreatic insulin delivery has not been investigated up to now. The purpose of the present investigation is to study the effects of these water-soluble nutrients and one of their esters (C12 triacylglycerol) on glucose-stimulated insulin release in isolated rat pancreatic islets.

\section{Materials and methods}

All tested medium-chain fatty acids were obtained from Sigma-Tau (Pomezia, Italy).

Pancreatic islets were isolated by the collagenase procedure (Malaisse-Lagae \& Malaisse, 1984) from female Wistar rats (B \& $\mathrm{K}$ Limited, Hull, E. Yorks., UK) given free access to food (KM-04-k12; Pavan Service, Oud Turnhout, Belgium) and tap water up to the time of killing by decapitation.

Insulin secretion was measured over a 90 min incubation at $37^{\circ} \mathrm{C}$ in groups of eight freshly isolated islets each placed in $1.0 \mathrm{ml}$ bicarbonate-buffered medium (Malaisse-Lagae \& Malaisse, 1984) containing bovine serum albumin $(5 \mathrm{mg} /$ $\mathrm{ml})$, D-glucose $(7 \cdot 0$ or $11.1 \mathrm{mM})$ and, as required, an aliphatic dioic acid or glycerol-1,2,3-tris(dodecanedioate) $(1 \cdot 0-10 \cdot 0 \mathrm{mM})$.

Since medium-chain fatty acids may interfere with the radioimmunoassay of insulin (Malaisse \& Malaisse-Lagae, 


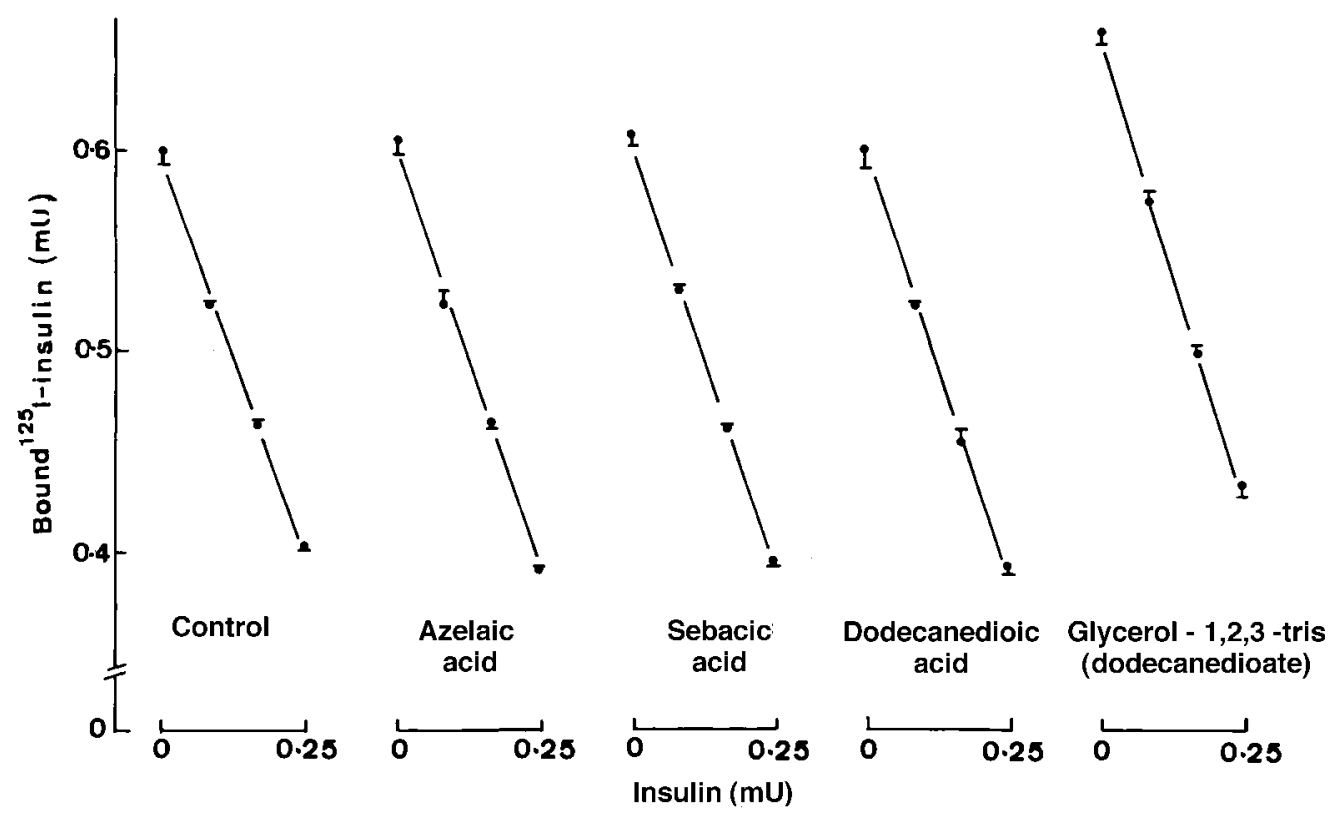

Fig. 1. Binding of ${ }^{125} \mathrm{I}$-insulin by guinea-pig anti-insulin serum, which was preincubated for $30 \mathrm{~min}$ at $37^{\circ} \mathrm{C}$ in the presence of increasing amounts of unlabelled insulin (abscissae), the assay medium containing, as required, azelaic acid, sebacic acid, dodecanedioic acid or glycerol-1,2,3tris(dodecanedioate) at the same final concentration as that used for measuring insulin release from islets incubated in the presence of $10.0 \mathrm{~mm}$ aliphatic dioic acids and $4.0 \mathrm{~mm}$ glycerol ester. Values are means with standard errors of the means for four estimations. For such a measurement, aliquots $(0.2 \mathrm{ml})$ of the incubation medium are mixed with $0.8 \mathrm{ml}$ phosphate buffer $(0.1 \mathrm{M}, \mathrm{pH} 7.0)$ containing diluted guinea-pig anti-insulin serum and, after $30 \mathrm{~min}$ incubation at $37^{\circ} \mathrm{C}$, exposed for another period of $30 \mathrm{~min}$ at $20^{\circ} \mathrm{C}$ to a mixture of unlabelled bovine and ${ }^{125} \mathrm{I}-$ labelled porcine insulin. Excess unbound insulin is then removed by adsorption to cellulose.

1968), control experiments were performed to assess the possible effect of four of the tested fatty acids on the backtitration procedure used for the measurement of insulin released by the islets (Wright et al. 1967). As shown in Fig. 1 , the results obtained in the presence of azelaic, sebacic and dodecanoic acids were undistinguishable from the control data. Glycerol-1,2,3-tris(dodecanedioate), however, increased the amount of bound ${ }^{125} \mathrm{I}$-insulin, without affecting either the blank value measured in the absence of guinea-pig anti-insulin serum (data not shown) or the decrease in ${ }^{125}$ I-insulin binding attributable to preincubation of the guinea-pig antibodies with unlabelled insulin (Fig. 1). In these control experiments, the concentration of the tested agents was identical to that used in the measurement of insulin release. Pooling all available data, the paired ratio of ${ }^{125} \mathrm{I}$-insulin binding in the presence or absence of glycerol-1,2,3-tris(dodecanedioate):guineapig antibodies preincubated in the absence of exogenous insulin averaged 108.8 (SEM 0.6) \% ( $n$ 5, $P<0.001$ as compared to unity).

All results, including those mentioned earlier, are expressed as means with standard errors of the means together with the number of individual observations $(n)$. The statistical significance of differences between mean values was assessed by use of Student's $t$ test.

\section{Results}

In the presence of $7.0 \mathrm{mM}$ D-glucose, both azelaic and sebacic acids (10.0 mM each) augmented insulin output
$(P<0.001)$, whereas dodecanedioic acid $(10 \cdot 0 \mathrm{~mm})$ failed to do so (Table 1). Comparable results were obtained at a higher concentration of D-glucose $(11.1 \mathrm{mM})$. Thus, azelaic acid $(10.0 \mathrm{mM})$ again augmented insulin secretion $(P<$ $0 \cdot 03$ ), whilst the increment in insulin output attributable to sebacic acid and dodecanedioic acid failed to achieve statistical significance.

When tested at lower concentrations (1.0 to $4.0 \mathrm{mM})$, azelaic acid, sebacic acid and dodecanedioic acid all failed to augment significantly insulin output evoked by $7.0 \mathrm{mM}$ D-glucose (Table 2). However, in this low range of concentrations, glycerol-1,2,3-tris(dodecanedioate) augmented glucose-stimulated insulin release. The most pronounced effect of the triacylglycerol was recorded at a concentration of $1.0 \mathrm{mM}$, when the output of insulin averaged 158.1 (SEM 14.3) \% ( $n$ 19, $P<0.005)$ of the mean corresponding control value (100.0 (SEM 11.1) \%; $n$ 20). In this second series of experiments, the release of insulin measured in the sole presence of $7.0 \mathrm{mM}$ D-glucose averaged $51.8($ SEM 7.1) $\mu \mathrm{U} /$ islet per $90 \mathrm{~min}(n 75)$.

In the last series of experiments, which were again conducted in the presence of $7.0 \mathrm{mM}-\mathrm{D}$-glucose, further aliphatic dioic acids with $3-14 \mathrm{C}$ atoms were tested in the range of 1.0-10.0 $\mathrm{mM}$ (Table 3 ). In some cases, the highest of these concentrations $(10 \cdot 0 \mathrm{mM})$ was not tested, because it had already been examined in prior publications (succinic acid; see Malaisse et al. 1993) or failed to yield a clear solution in the bicarbonate-buffered medium (tetradecanedioic acid). In this last set of experiments, the secretion of insulin recorded in the sole presence of D-glucose averaged 48.8 (SEM 3.5) $\mu \mathrm{U} /$ islet per $90 \mathrm{~min}$ ( $n$ 123). Malonic acid, 
Table 1. Effects of azelaic acid, sebacic acid and dodecanedioic acid on insulin release evoked by 7.0 and 11.1 mM-D-glucose*

(Mean values with standard errors of the means)

\begin{tabular}{|c|c|c|c|c|c|c|}
\hline \multirow[b]{3}{*}{ Tested agent } & \multicolumn{6}{|c|}{ Insulin output ( $\mu \mathrm{U} /$ islet per $90 \mathrm{~min})$} \\
\hline & \multicolumn{3}{|c|}{ D-glucose $(7.0 \mathrm{mM})$} & \multicolumn{3}{|c|}{ D-glucose(11.1 mM) } \\
\hline & Mean & SEM & $n$ & Mean & SEM & $n$ \\
\hline Nil & $41 \cdot 1$ & 3.8 & 24 & 247.9 & $17 \cdot 6$ & 24 \\
\hline Azelaic acid (10.0 mM) & $66 \cdot 8$ & $5 \cdot 9$ & 24 & 301.5 & $15 \cdot 2$ & 24 \\
\hline Sebacic acid (10.0 mM) & $67 \cdot 9$ & 4.5 & 23 & $271 \cdot 3$ & $11 \cdot 6$ & 24 \\
\hline Dodecanedioic acid $(10.0 \mathrm{~mm})$ & $46 \cdot 3$ & 3.4 & 24 & 271.6 & $16 \cdot 9$ & 24 \\
\hline
\end{tabular}

${ }^{*}$ For details of procedures see p. 733.

succinic acid, pimelic acid and undecanedioic acid all failed to affect insulin release significantly. Tridecanedioic acid augmented glucose-stimulated insulin release $(P<$ 0.05 ) when tested at a $10.0 \mathrm{mM}$ concentration, but not at lower concentrations. Only tetradecanedioic acid inhibited $(P<0.005$ or less) the secretory response to D-glucose.

\section{Discussion}

The role of the interaction between glucose metabolism and intracellular fatty acids disposition in coupling stimulation and secretion of insulin from $\beta$-cells has been largely recognized (Greenough et al. 1967; Malaisse \& MalaisseLagae, 1968; Campillo et al. 1979; McGarry \& Dobbins, 1999). A common element in the glucose-fatty acid metabolism pathways is the malonyl-CoA molecule (Ruderman et al. 1999), whose concentration is thought to modulate the access of these energy substrates to the Krebs cycle.

Glycolysis allows the formation of pyruvate, which can be converted to citrate in mitochondria. While a fraction of this citrate is oxidized in the tricarboxylic-acid cycle to $\mathrm{CO}_{2}$ and $\mathrm{H}_{2} \mathrm{O}$ generating ATP, the remainder can leave the mitochondria to be converted into malonyl-CoA.

The increase in malonyl-CoA cytosolic level, by suppressing the carnitine palmitoyltransferase I activity, determines an increase in the cytosolic concentration of long-chain acyl-CoA. This last event may act as a signal for insulin secretion.

The $\beta$-oxidation of one mol odd-numbered $\mathrm{C}$ atom dicarboxylic acid produces, together with a certain number of acetyl-CoA mol proportional to the dicarboxylic acid chain length, one mol malonyl-CoA, while even-numbered dicarboxylic acids allow formation of succinyl-CoA in addition to acetyl-CoA. It is likely, therefore, that oddnumbered dicarboxylic acids can stimulate insulin release from pancreatic $\beta$-cells in the presence of glucose. We indeed observed that, in presence of $7.0 \mathrm{mM}$-D-glucose, azelaic acid (C9) causes a $62 \%$ increase in insulin output at a concentration of $10 \mathrm{mM}$, while this effect is diminished to about half $(35 \%)$ as its concentration is reduced ten-fold (1 mM). The effect of sebacic acid (C10) was similar to that of $\mathrm{C} 9$ at concentrations of $10 \mathrm{mM}$, while at $1 \mathrm{mM} \mathrm{C} 10$ increased insulin output by only $17 \%$; such an increase failed to achieve statistical significance. Among the other nutrients tested, $1 \mathrm{mM}$ glycerol triester of dodecanedioic acid produced the largest stimulation of $\beta$-cell insulin release (58\% greater than control).

The similar effect of C9 and C10 suggests that both malonyl-CoA and succinyl-CoA, which are the end-products of the $\beta$-oxidation of these two dicarboxylic acids respectively, produce a quantitatively similar stimulatory effect on insulin release. These results are also in accordance with previous observations (Malaisse et al. 1993; Ladrière et al. 1999) that the esters of succinic acid show a high insulinotropic efficiency both in vitro and in vivo.

It may appear surprising that dodecanedioic acid (C12), which has the same metabolic pathway as $\mathrm{C} 10$, did not show the same degree of effect when tested at a $10 \mathrm{~mm}$ concentration. This might be explained by the different water solubility of the two diacids, which implies that at this concentration a lower amount of $\mathrm{C} 12$ can enter the $\beta$-cells to be $\beta$-oxidized. In fact, at a $1.0 \mathrm{~mm}$ concentration the effects of $\mathrm{C} 10$ and C12 were comparable. Tetradecanediodic acid inhibited glucose-stimulated insulin release. The determinants of this unexpected situation remain to be elucidated.

As far as the triacylglycerol of $\mathrm{C} 12$ is concerned, the higher stimulatory effect on insulin release at $1 \mathrm{mM}$

Table 2. Effects of low concentrations of azelaic acid, sebacic acid, dodecanedioic acid and its ester on insulin release evoked by 7.0 mM-Dglucose*

(Mean values with standard errors of the means)

\begin{tabular}{|c|c|c|c|c|c|c|c|c|c|}
\hline \multirow[b]{2}{*}{ Diacid (ester) } & \multicolumn{3}{|c|}{$0.0 \mathrm{~mm}$} & \multicolumn{3}{|c|}{$1.0 \mathrm{~mm}$} & \multicolumn{3}{|c|}{$4.0 \mathrm{mM}$} \\
\hline & Mean & SEM & $n$ & Mean & SEM & $n$ & Mean & SEM & $n$ \\
\hline Azelaic acid & $100 \cdot 0$ & $20 \cdot 7$ & 15 & $135 \cdot 0$ & $26 \cdot 3$ & 18 & $134 \cdot 0$ & $17 \cdot 4$ & 19 \\
\hline Sebacic acid & $100 \cdot 0$ & $14 \cdot 6$ & 16 & $117 \cdot 5$ & $15 \cdot 3$ & 18 & $111 \cdot 0$ & $15 \cdot 0$ & 18 \\
\hline Dodecanedioic acid & $100 \cdot 0$ & 8.0 & 24 & 113.5 & $11 \cdot 1$ & 24 & 114.3 & $9 \cdot 0$ & 24 \\
\hline Glycerol-1,2,3-tris(dodecanedioate) & $100 \cdot 0$ & $11 \cdot 1$ & 20 & $158 \cdot 1$ & $14 \cdot 3$ & 19 & $132 \cdot 6$ & $9 \cdot 9$ & 19 \\
\hline
\end{tabular}

${ }^{\star}$ All results are expressed as a percentage of the mean value recorded within the same experiment(s) in the sole presence of $D$-glucose. For details of procedures see p. 733. 
Table 3. Effects of aliphatic dioic acids on insulin release evoked by $7.0 \mathrm{mM}$-D-glucose* (Mean values and standard errors of the means)

\begin{tabular}{|c|c|c|c|c|c|c|c|c|c|c|c|c|c|c|c|}
\hline & \multicolumn{3}{|c|}{$0.0 \mathrm{~mm}$} & \multicolumn{3}{|c|}{$1.0 \mathrm{~mm}$} & \multicolumn{3}{|c|}{$2.0 \mathrm{~mm}$} & \multicolumn{3}{|c|}{$4.0 \mathrm{~mm}$} & \multicolumn{3}{|c|}{$10 \cdot 0 \mathrm{~mm}$} \\
\hline & Mean & SEM & $n$ & Mean & SEM & $n$ & Mean & SEM & $n$ & Mean & SEM & $n$ & Mean & SEM & $\bar{n}$ \\
\hline Malonic acid & $100 \cdot 0$ & 28.4 & 17 & 94.1 & 14.8 & 19 & $72 \cdot 1$ & $10 \cdot 2$ & 19 & 91.8 & 17.9 & 14 & 111.5 & $15 \cdot 9$ & $\overline{18}$ \\
\hline Succinic acid & $100 \cdot 0$ & $25 \cdot 6$ & 15 & $114 \cdot 2$ & $19 \cdot 3$ & 17 & 84.5 & $25 \cdot 9$ & 18 & $76 \cdot 1$ & $20 \cdot 0$ & 18 & & ND & \\
\hline Pimelic acid & $100 \cdot 0$ & 8.7 & 19 & 113.6 & $10 \cdot 1$ & 18 & $105 \cdot 8$ & 9.0 & 20 & 95.5 & 9.0 & 20 & & ND & \\
\hline Undecanedioic acid & $100 \cdot 0$ & $12 \cdot 5$ & 24 & 93.2 & $10 \cdot 9$ & 24 & 99.5 & 7.9 & 24 & $115 \cdot 8$ & $9 \cdot 2$ & 24 & $100 \cdot 5$ & 11.4 & 24 \\
\hline Tridecanedioic acid & $100 \cdot 0$ & $12 \cdot 1$ & 24 & 86.6 & 9.9 & 24 & $105 \cdot 8$ & $20 \cdot 6$ & 24 & 95.9 & 9.6 & 24 & 141.4 & $15 \cdot 3$ & 24 \\
\hline Tetradecanedioic acid & $100 \cdot 0$ & $10 \cdot 0$ & 24 & $52 \cdot 2$ & $6 \cdot 3$ & 24 & $48 \cdot 8$ & $12 \cdot 1$ & 24 & $28 \cdot 1$ & 3.5 & 24 & & ND & \\
\hline
\end{tabular}

ND, not determined.

* All results are expressed as a percentage of the mean value recorded within the same experiment(s) in the sole presence of $D$-glucose. For details of procedures see p. 733.

concentration might be ascribed to a bypass phenomenon of dicarboxylic acid-specific receptors with a passive transport by diffusion across the cellular membrane, like that hypothesized for glucose esters (Malaisse, 1999). It is also likely that, once one molecule $\mathrm{C} 12$ triacylglycerol enters the $\beta$-cells, it must be hydrolysed to one molecule glycerol and three molecules dodecanedioic acid. The higher amount of $\mathrm{C} 12$ supplied to the cells could thus explain the higher stimulatory effect on insulin release.

In conclusion, the present study showing a direct effect of dicarboxylic acids on insulin-producing cells further supports the contention that medium-chain dicarboxylic acids can be regarded as useful energy substrates. This holds true also for glycerol-1,2,3-tris(dodecanedioate), at least when this triacylglycerol is used at a low concentration. These findings are obviously relevant to the possible use of these alternative nutrients in clinical situations, such as decompensated diabetes mellitus (Greco \& Mingrone, 1995; Mingrone et al. 1997).

\section{Acknowledgements}

This study was supported by a grant (3.4513.94) from the Belgian Foundation for Scientific Medical Research. We are grateful to N. Bolaky and M. Mahy for technical assistance, and C. Demesmaeker for secretarial help.

\section{References}

Bertuzzi A, Gandolfi A, Salinari S, Mingrone G, Arcieri-Mastromattei E, Finotti E \& Greco AV (1991) Pharmacokinetic analysis of azelaic acid disodium salt: a proposed substrate for total parenteral nutrition. Clinical Pharmacokinetics 20, 411-419.

Campillo JE, Luyckx AS, Torres MD \& Lefebvre PJ (1979) Effect of oleic acid on insulin secretion by the isolated perfused rat pancreas. Diabetologia 16, 267-273.

De Gaetano A, Mingrone G, Castagneto M, Benedetti G, Greco AV \& Gasbarrini G (1999) Kinetics of dodecanedioic acid triglyceride in rats. American Journal of Physiology 276, E497-E502.

Greco AV \& Mingrone G (1995) Dicarboxylic acids, an alternate fuel substrate in parenteral nutrition: an update. Clinical Nutrition 14, 143-148.

Greenough WB, Crespin SR \& Steinberg D (1967) Hypoglycemia and hyperinsulinemia in response to raised free fatty acid levels. Lancet ii, 1334-1336.
Ladrière L, Björkling F \& Malaisse WJ (1999) Assessment of the nutritional value of glycerol-1,2,3-tris(methylsuccinate) in fed and starved rats. Molecular Genetics and Metabolism 3, 254-260.

McGarry JD \& Dobbins RL (1999) Fatty acids, lipotoxicity and insulin secretion. Diabetologia 42, 128-138.

Malaisse WJ (1999) Insulinotropic action of monosaccharide esters: therapeutic perspectives. Diabetologia 42, 286-291.

Malaisse WJ \& Malaisse-Lagae F (1968) Stimulation of insulin secretion by non-carbohydrate metabolites. Journal of Laboratory and Clinical Medicine 72, 438-448.

Malaisse WJ, Rasschaert J, Villanueva-Peñacarrillo MM \& Valverde I (1993) Respiratory, ionic and functional effects of succinate esters in pancreatic islets. American Journal of Physiology 264, E428-E433.

Malaisse-Lagae F \& Malaisse WJ (1984) Insulin release by pancreatic islets. In Methods in Diabetes Research, pp. 147152 [J Larner and SL Phol, editors]. New York, NY: Wiley.

Mingrone G, De Gaetano A, Greco AV, Benedetti G, Capristo E, Castagneto M \& Gasbarrini G (1996) Plasma clearance and oxidation of dodecanedioic acid in humans. Journal of Parenteral and Enteral Nutrition 20, 38-42.

Mingrone G, De Gaetano A, Greco AV, Capristo E, Benedetti G, Castagneto M \& Gasbarrini G (1997) Dodecanedioic acid infusion induces a sparing effect on whole body glucose uptake, mainly in non-insulin-dependent diabetes mellitus. British Journal of Nutrition 78, 723-735.

Mingrone G, Tacchino RM, Castagneto M, Finotti E \& Greco AV (1992) Use of even-numbered carbon atom dicarboxylic salts in parenteral nutrition as fuel substrate. Journal of Parenteral and Enteral Nutrition 16, 32-38.

Mingrone G, Tacchino RM, Greco AV, Arcieri-Mastromattei E, Marino F \& Castagneto M (1989) Preliminary studies of a dicarboxylic acid as an energy substrate in man. Journal of Parenteral and Enteral Nutrition 13, 299-305.

Raguso C, Mingrone G, Greco AV, Tataranni PA, De Gaetano A \& Castagneto M (1994) Dicarboxylic acids and glucose utilization in humans: effect of sebacate. Journal of Parenteral and Enteral Nutrition 18, 9-13.

Ruderman NB, Saha AK, Vavvas D \& Witters LA (1999) Malonyl-CoA, fuel sensing, and insulin resistance. American Journal of Physiology 276, E1-E18.

Tacchino RM, Mingrone G, Marino F, Arcieri-Mastromattei E \& Greco AV (1990) Short term infusion of azelaic acid versus intralipid in healthy subjects evaluated by indirect calorimetry. Journal of Parenteral and Enteral Nutrition 14, 169-175.

Wright PH, Malaisse WJ \& Reynolds IJ (1967) The assay of partially neutralized guinea-pig anti-insulin serum. Endocrinology 81, 226-234. 